\title{
More awareness is needed for severe acute respiratory syndrome coronavirus 2019 transmission through exhaled air during non-invasive respiratory support: experience from China
}

To the Editor:

As of 17 February, 2020, China has 70635 confirmed cases of coronavirus disease 2019 (COVID-19), including 1772 deaths [1]. Human-to-human spread of the virus via respiratory droplets is currently considered to be the main route of transmission. However, exhaled air dispersion during non-invasive respiratory support may increase the risk of coronavirus transmission, and requires more attention from medical personnel and patients.

According to the Chinese Centre for Disease Control and Prevention [2], among 72314 reported cases in mainland China (44672 confirmed cases, 16186 suspected cases, 10567 clinically diagnosed cases, and 889 asymptomatic infected cases) as of 11 February, 1716 medical staff were infected. Notably, WANG et al. [3] analysed the clinical characteristics of 138 hospitalised cases with COVID-19 and found that the nosocomial infection rate in the study was about $41.3 \%$, with 17 inpatients and 40 medical staff. Given the high use of respiratory support to treat dyspnoea and respiratory failure induced by COVID-19, viral transmission through exhaled air should be considered.

When oxygen is delivered through nasal catheter, mask or non-invasive ventilation (NIV), substantial exhaled air is released into the air, which can increase dispersion of the virus, and subsequently increase the risk of nosocomial infection [4]. Prior studies have suggested that the application of high-flow nasal cannula (HFNC), NIV through the specific mask with optimised vent holes or the helmet with a double-limb circuit may lower the risk of airborne transmission [5, 6]. Performing non-invasive respiratory support in a single, well-ventilated, negative pressure ward is also considered a safe option. Currently, however, the majority of patients are still receiving respiratory support through nasal catheter or common mask in general wards or emergency departments with limited medical resources. The potential for airborne transmission in this population has not received enough attention. Furthermore, a significant proportion of suspected patients with mild to no symptoms who are managing at home may also require long-term home oxygen or NIV treatment (e.g. patients with advanced staged COPD). These patients may increase the risk of family cluster infections by the widespread dispersion of exhaled air in their homes.

Non-invasive respiratory support plays an essential role in the treatment of COVID-19, and more awareness is needed regarding the increased risk of viral transmission from exhaled air. Medical staff should utilise personal protective equipment when providing respiratory support therapy. Patients should wear a medical mask when receiving conventional oxygen therapy or HFNC in order to reduce air dispersion. When performing NIV therapy, because helmet resources are limited and they are not frequently used in routine clinical practice, we suggest avoiding masks with vent holes and adding a filter between the mask and the vent valve to reduce viral transmission. Patient beds should be at least $1 \mathrm{~m}$ from one another and the ward air circulation rate needs to be increased [7]. For patients with suspected COVID-19 infection receiving long-term respiratory support at home, it is recommended that they stay in a single, well-ventilated room to avoid potentially infecting their family members.

@ERSpublications

Non-invasive respiratory support plays an essential role in the treatment of COVID-19. More awareness is needed of viral transmission through exhaled air. http://bit.ly/39kNrrF

Cite this article as: Guan L, Zhou L, Zhang J, et al. More awareness is needed for severe acute respiratory syndrome coronavirus 2019 transmission through exhaled air during non-invasive respiratory support: experience from China. Eur Respir J 2020; 55: 2000352 [https://doi.org/10.1183/13993003.00352-2020]. 
Lili Guan $^{1,5}$, Luqian Zhou ${ }^{1,5,6}$, Jinnong Zhang ${ }^{2,5}$, Wei Peng ${ }^{3,5}$ and Rongchang Chen ${ }^{1,4,6}$

${ }^{1}$ State Key Laboratory of Respiratory Disease, National Clinical Research Center for Respiratory Disease, Guangzhou Institute of Respiratory Health, The First Affiliated Hospital of Guangzhou Medical University, Guangzhou, China. ${ }^{2}$ Dept of Emergency Medicine, Union Hospital, Tongji Medical College, Huazhong University of Science and Technology, Wuhan, China. ${ }^{3}$ Division of ICU and Respiratory Service, Dept of Internal Medicine, Salt Lake Regional Medical Center, Heart and Lung Institute of Utah, Salt Lake City, UT, USA. ${ }^{4}$ Dept of Respiratory and Critical Care Medicine, First Affiliated Hospital of Southern University of Science and Technology, Second Clinical Medical College of Jinan University, Shenzhen People's Hospital, Shenzhen Institute of Respiratory Diseases, Shenzhen, China. ${ }^{5}$ These authors contributed equally to this work as first authors. ${ }^{6}$ These authors contributed equally to this work as senior authors.

Correspondence: Rongchang Chen, Dept of Respiratory and Critical Care Medicine, First Affiliated Hospital of Southern University of Science and Technology, Second Clinical Medical College of Jinan University, Shenzhen People's Hospital, Shenzhen Institute of Respiratory Diseases, 1017 Dong Men Road, Shenzhen 518020, China. E-mail: chenrc@vip.163.com

Received: 18 Feb 2020 | Accepted: 22 Feb 2020

Conflict of interest: None declared.

\section{Reference}

1 World Health Organization. Novel Coronavirus (2019-nCoV). Situation Report-28 Feb 17, 2020. www.who.int/ docs/default-source/coronaviruse/situation-reports/20200217-sitrep-28-covid-19.pdf?sfvrsn=a19cf2ad_2 Date last updated: 17 February, 2020. Date last accessed: 17 February 2020.

2 The Novel Coronavirus Pneumonia Emergency Response Epidemiology Team. [The epidemiological characteristics of an outbreak of 2019 novel coronavirus diseases (COVID-19) in China] (in Chinese). Zhonghua Liu Xing Bing Xue Za Zhi 2020; 41: 145-151.

3 Wang $\mathrm{D}, \mathrm{Hu} \mathrm{B}, \mathrm{Hu}$ C, et al. Clinical characteristics of 138 hospitalized patients with 2019 novel coronavirus-infected pneumonia in Wuhan, China. JAMA 2020; in press [https://doi.org/10.1001/jama.2020.1585].

4 Simonds AK, Hanak A, Chatwin M, et al. Evaluation of droplet dispersion during non-invasive ventilation, oxygen therapy, nebuliser treatment and chest physiotherapy in clinical practice: implications for management of pandemic influenza and other airborne infections. Health Technol Assess 2010; 14: 131-172.

5 Hui DS, Chow BK, Lo T, et al. Exhaled air dispersion during high-flow nasal cannula therapy versus CPAP via different masks. Eur Respir J 2019; 53: 1802339.

6 Hui DS, Chow BK, Lo T, et al. Exhaled air dispersion during noninvasive ventilation via helmets and a total facemask. Chest 2015; 147: 1336-1343.

7 World Health Organization. Infection Prevention and Control during Health Care when Novel Coronavirus (nCoV) Infection is Suspected. www.who.int/publications-detail/infection-prevention-and-control-during-health-care-whennovel-coronavirus-(ncov)-infection-is-suspected-20200125 Date last updated: 25 January, 2020. Date last accessed: 17 February, 2020. 